\title{
Ueber die korrelativen Beziehungen zwischen dem Nucleus caudatus, dem Putamen und dem Globus pallidus zueinander.
}

\author{
Von \\ Dr. Saburo Inui. \\ Aus dent anatomischen Institut der Universitat Okayama, \\ (Vorstand: Prof. Dr. K. Kosaka). \\ Eingegnngen nm 25. November 1927.
}

Das ist bis jetzt zwar vielfach zum Gegenstand der Untersuchung geworden, und nicht wenige Autoren haben sich damit beschäftigt. Was aber die Resultate angeht, so stehen die Ansichten schroff gegenüber, so dass es noch weiterer Untersuchungen bedarf, um das betreffende Problem endgültig zu entscheiden. Dies gilt vor allem für die Frage, ob die beiderseitigen Corpora striata miteinander in Verbindung stehen. Dazu kommt noch, dass die bisherigen Untersuchungen ausschliesslich nach der Karminfärbung oder nach dem Marchi-Verfahren vorgenommen wurden, wâs mich immer dazu zwingt, am Káninchenkopf einige Fxperimente anzustellen. So habe ich erstens den Nucleus caudatus, zweitens das Putamen und drittens den Globus pallidus zerstort, um das abgetragene Gehirn an der Hand der Nissl'schen und der Marchi'schen Methode zu untersuchen. Natürlich bleibt es an meiner Untersuchung noch viel zu wünschen übrig, so dass sie noch lange nicht für abgeschlossen gehalten werden kann. Indessen habe ich mich entschlossen, die bereits von mir experimentell festgestellten Tatsachen hier mitzuteilen, da sie nicht ohne Interesse sind.

Es scheint mir am Platze zu sein, die einschlägige Literatur vorauszuschicken, bevor ich zur Erwähnung meiner eigenen Untersuchung übergehe.

\section{Literatur.}

Nach Zerstörung des ventro-medialen Teils der bilateralen Nuclei caudati bei der Katze konstatiert Probst, dass die degenerierten Fasern aus der Verletzungsstelle durch die Capsula interna hindurch den Linsenkern erreichen, um darin blind zu endigen. Pollak gibt in seiner Publikation diejenigen Fasern an, welche aus dem Nucleus caudatus entepringen und im Globus pallidus endigen. Nach Grünstein sind die strio-pallidären 
Fasern beim Hunde teils fein, teils dick. Die feinen Fasern durchkreuzen zu einem Bündel zusammentretend die Capsula interna, während die dicken durch die Capsula interna hindurch in die Lamina medullaris externa eintreten. Die beiden Fasern strahlen endlich radiär in den Globus pallidus ein, was auch für diejenigen der Fall ist, welche bei Verletzung des Putamens in Degeneration verfallen. Ebenso nimmt Minkowsky die hauptsächliche Endigung der aus Nucleus caudatus und Putamen entspringenden Fasern im Globus pallidus an und es gelingt ihm ferner, vom Globus pallidus gegen das Putamen hinziehende Fasern zu konstatieren.

Nach Vogt ist die Pars externa des Globus pallidus von denjenigen Bündeln zusanmengesetzt, welche teils aus dünnen strio-pallidären, teils aus etwas dickeren striopetalen Fasern bestehen. Der Autor äussert sich dahin aus, dass im Corpus striatum die aus den kleinen Zellen entspringenden Fasern um die grossen perizellulär endigen, und die letzteren ihrerseits den strio-pallidären Fasern zum Ursprung dienen. Bezüglich der strio-pallidären Fasern sagt Jakob auch, dass sie sämtlich aus den grossen Zellen des Corpus striatum stammen und im Globus pallidus endigen. Nach ihm sollen die Marklamellen des Globus pallidus vornehmlich aus denjenigen Fasern bestehen, welche entweder dem Thalamus entstammend im Corpus striatum endigen, oder aus dem Corpus striatum entspringend in den Globus pallidus eintreten. Der Autor ist der Meinung, dass die aus dem Nucleus caudatus stammenden Fasern wenigstens zum Teil durch den Globus pallidus hindurch laufen, tum den Thalamus zu erreichen.

Was die Verbindung des Nucleus caudatus mit Putamen und Gilobus pallidus angeht, so darf man die Ansicht von Wilson nicht ausser Acht lassen, wonach die aus dem Nucleus caudatus entspringenden Fasern hauptsächlich im Putamen endigen; nicht wenige von den Fasern laufen aber am seitlichen Teil des Globus pallidus vorbei, während die aus Putamen und Globus pallidus stammenden Fasern in den Nucleus caudatus eintreten. Leider gelingt es Wilson nicht, beim Affen derjenigen Fasern gewahr zu werden, welche die beiderseitigen Corpora striata miteinander verbinden. Die Verbindung der genannten Körper miteinander soll nach Valkenburg durch das Corpus callosum vermittelt werden.

Nach der Vorausschickung der Literatur werde ich nun zur Erwähnung meiner eigenen Untersuchung übergehen, welche in folgenden 3 Experimenten besteht.

\section{Vereuch I. Zerstörung des Nucleus caudatus.}

Zur Operation bedient man sich einer spitzen Nadel, welche unmittelbar lateral von der Fissura longitudinalis ins kaudale Ende des Frontallappens gesteckt wird. In ventro-lateraler Richtung durch die Hirnrinde hindurch geht man in die Tiefe und 
zerstort so den Kopf des rechten Nucleus caudatus. Diesem operativen Eingriffe wurden im Ganzen 4 Kaninchen unterworfen, unter denen 2 am 14. Tage post operationem getotet wurden, während die übrigen 21 Tage die Operation überlebten. Die aus den ersteren stammenden Gehirne waren in $20 \mu$ dicke Frontalschnitte zerlegt, deren Färbung mit Thionin geschah. Dagegen wurden die Gehirne, welche aus den am 21. Tage post operationem getoteten Tieren abgetragen waren, nach dem Marchi-Verfahren untersucht.

Beim Durchmustern der nach dem Nissl-Verfahren gefärbten Schnitte findet man zunächst, dass im Niveau des mittleren Drittels des Septum pellucidum nicht nur das Stratum sagittale mit dem Balken, sondern auch der Kopf des Nucleus caudatus stark zerstort sind. Die Wunde ist schräg von dorso-medial nach ventro-lateral gerichtet und zeigt sich am Stratum sagittale keilformig, am Kopfe des Nucleus caudatus bandformig. Etwas kaudaler greift sie den mittleren Teil des Nucleus caudatus an, wobei auch der daran sich anschliessende Teil der Capsula interna teilweise in Mitleidenschaft gezogen ist (Fig. 1).

Mikroskopisch tritt die lädierte Stelle des Nucleus caudatus bedeutend blass auf und schliesst mehr keine Ganglienzellen in sich. Sie ragt stark in den Seitenventrikel hervor, und man findet in ihrer Umgebung eine mässige Wucherung des Gliagewebes. Kaudalwärts hin nimmt sie ziemlich plotzlich an Umfang ab und verliert sich ungefähr im Niveau des kaudalen Endes des Septum pellucidum. Der Nucleus caudatus erweist sich in einer an die Wunde unmittelbar sich anschliessenden Zone so stark als pathologisch verändert, dass hier die Ganglienzellen meistens spurlos verschwunden, und übrig bleibende auch in hochgradige Degeneration verfallen (Fig. 1, dl u. vm). Dorsal von der lädierten Stelle begegnet man einer Anzahl degenerierter Ganglienzellen, welche eine mehr oder weniger deutliche Aufblähung des Zellkorpers, eine tiefgreifende Zerstäubung der Nissl'schen Korper und eine exzentrische Verlagerung des Zellkernes aufweisen. Obgleich die grossen Zellen ebenfalls auch ventral von der lädierten Stelle degeneriert gefunden werden, so treten doch die obigen Degenerationsbilder hier nicht so deutlich zutage, wie dorsal von der lädierten Stelle. Im Gegensatz zu den grossen Zellen bleiben die kleinen, seien sie dorsal oder ventral von der lädierten Stelle angetroffen, immer intakt. Im Niveau, wo der Nucleus caudatus am stärksten verletzt ist, erweisen sich einige von den grossen Zellen auch im kontralateralen Kern als verändert, während die kleinen auch hier von der degenerativen Veränderung verschont bleiben.

Was die Nissl-Degeneration im Putamen angeht, so wird sie auf beiden Seiten sowohl an den grossen, als auch an den kleinen Zellen angetroffen, nur dass die degenerierten Zellen auf der nicht operierten Seite viel weniger zahlreich sind, als auf der operierten. Hier erweisen sich die grossen Zellen zum grossen Teil als verändert, und der Veränderungsgrad ist fast derselbe, wie im Nucleus caudatus (Fig 2, Dgr Z). Wenn auch die degenerierten kleinen Zellen im Verhältnis zu den normalen nur äusserst spärlich sind, 
so sind sie doch häufig so stark degeneriert, dass sie ganz blasig aussehen (Fig. 2, Dkl Z). Man begegnet der Nissl-Degeneration hauptsächlich im Niveau, wo die Wunde des Nucleus caudatus gefunden wịd. Kranial und kaudal davon gibt es zwar veränderte Zellen; ihre Zahl ist aber so gering, dass es gleichgültig ist, ob man sie in Anschlag bringt oder nicht.

Bei Zerstorung des Nucleus caudatus sind die Zellen im Globus pallidus viel zahlreicher verändert, und die Degeneration tritt viel stärker zutage, als im Putamen. So geraten die Zellen dort meistens in Degeneration und bieten eine hochgradige Aufblähung des Zellkorpers, eine fast vơllige Auflosung der Nissl'schen Korper und das Versohwinden des Zellkernes dar (Fig. 3, DZ). Auch auf der nicht operierten Seite sind die degenerierten Zellen anzutreffen; aber ihre Zahl ist hier viel geringer, als auf der operierten.

Wie oben erwähnt, ist bei dem Experimente der Kopf des Nucleus caudatus von einer schräg von dorso-lateral nach ventro-medial gerichteten Wunde stark befallen, wobei auch die Capsula interna leicht mitlädiert ist. Bei der Mitleidenschaft der inneren Kapsel fragt es sich zwar, ob die im Putamen sich abspielende Degeneration entweder auf die Wunde des Nucleus caudatus, oder auf die Verletzung der inneren Kapsel zurückzuführen ist. Die letztere ist aber zu leicht, als dass sie hierbei in Anspruch genommen werden kann. Dazu kommt noch, dass die innere Kapsel dicht am Nucleus caudatus verletzt ist. Bei dieser Sachlage ist man schon berechtigt anzunehmen, dass die im Putamen gefundene Degeneration ihren Grund nicht in der Durchtrennung der aus dem Putamen entspringend durch die innere Kapsel hindurch gegen andere Hirnteile hinziehenden Axonen, sondern wohl in der Verletzung des Nucleus caudatus selbst hat. Ebenso ist die Degeneration im Globus pallidus auf die Verletzung des Nucleus caudatus zurückzuführen.

Hierbei sei hinzugefügt, dass ich in meiner vorigen Mitteilung angab, dass die Verletzung der Substantia nigra eine schwache Veränderung an den Zellen des Globus pallidus zur Folge hatte; ein Befund, welchèr mich damals zur Annahme nötigte, dass es den Zellen des Globus pallidus eigen sei, bei der Verletzung nur schwach sich zu verändern. Jetzt erkenne ich jedoch, dass die Zellen des Globus pallidus bei Zerstörung des Nucleus caudatus in hochgradige Degeneration verfallen.

Der Befund an den Gehirnen, welche aus den 21 Tage die Operation überlebten Tieren abgetragen und nach dem Marchi-Verfahren untersucht sind, lautet wie folgt: Was in erster Linie die durch die Operation bedingte Verletzung angeht, so ist im Niveau des kranialen Teiles des Septum pellucidum der Balken mit dem Stratum sagittale von einer schräg von dorso-medial nach ventro-lateral gerichteten Wunde so stark angegriffen, dass die Wunde sich in den Seitenventrikel weit offnet. Dabei ist auch der Kopf des Nucleus caudatus an seinem dorsalen Teil stark mitverletzt (Fig. 4). Etwas kaudal im Niveau des mittleren Drittels des Septum pellucidum wird die Wunde im Stratum 
sagittale schon kaum sichtbar, während sie im Balken noch deutlich zutage tritt und bis zur Capsula interna gelangt, ohne jedoch sie zu beschädigen. Kaudalwärts hin wird die Wunde des Nucleus caudatus immer kleiner und verliert sich endlich im Niveau des kranialen Endes des Ammonshorns. Über die Marchi-Degeneration ist zuerst anzugeben, dass im Niveau des kranialen Endes des Septum pellucidum medial von der Wunde des Balkens nur spärliche feine degenerierte Fasern sich finden, während lateral von derselben zahlreiche, ziemlich grobe degenerierte anzutreffen sind. Die degenerierten Fasern ziehen nach leteralwärts hin und treten teils in die innere, teils in die äussere Kapsel hinein. So kann man in dem ventralen Teil der letzteren sowie in dem daran sich anschliessenden Putamenteil zahlreiche feine degenerierte Fasern finden; ein Befund, welcher ohne Zweifel darauf hinweist, dass die in der äusseren Kapsel wahrgenommenen degenerierten Fasern ins Putamen eintreten. Auch in denjenigen Faserbündeln, welche von der inneren Kapsel sich teilend im Putamen sich zersplittern, wird die MarchiDegeneration beobachtet.

Im Nucleus caudatus finden sich die degenerierten Fasern dorsal von der lädierten Stelle viel zahlreicher, als ventral von derselben, und die dorsal von der verletzten Stelle angetroffenen degenerierten Fasern erreichen zum Teil durch die Capsula interna hindurch das Putamen, welches auch in seinem dorso-medialen Teil feine degenerierte Fasern enthält. Ebenso fallen diejenigen radiären Bündel, welche von der Verletzungsstelle ventro-lateral gegen die innere Kapsel hinziehen, der Degeneration anheim (Fig. 4).

Was im Globus pallidus befindliche degenerierte Fasern angeht, so stammen sie zum Teil aus dem Balken und gelangen durch die innere Kapsel hindurch zum medialen Teil des Globus pallidus, während der andere Teil der genannten Fasern aus dem Nucleus caudatus entspringend den lateralen Teil des Globus pallidus erreichen und darin sich zersplittern.

Auf der nicht operierten Seite kann man die degenerierten Fasern zwar nicht in Nucleus caudatus und Putamen konstatieren, wohl aber in dem Globus pallidus.

Aus dem oben gesagten kann man den Schluss ziehen, dass die aus dem Nucleus caudatus entspringenden Fasern durch die Capsula interna hindurch laufen, um teils ins Putamen, teils in den Globus pallidus einzutreten.

\section{Versuch II. Zerstörung des Putamens.}

Nachdem ich den Schädelknochen an der rechten Frontalgegend mittels eines kleinen Trepan̈s angebohrt hatte, ftihrte ich eine spitze Nadel in ventro-lateraler Richtung in die Wunde ein, um das rechte Putamen zu zerstören. Die Operation wurde an 6 Kaninchen angestellt, von denen 3 am 13. Tage post operationem getotet wurden, während die übrigen 21 Tage die Operation überlebten. Die Untersuchung des Gehirns wurde 
bei den ersteren Tieren nach der Nissl-Methode, bei den letzteren aber nach dem Marchi-Verfahren vorgenommen. Auch hier habe ich zuerst den Nissl'schen Befund zu erwähnen.

Wenn man die Nissl'schen Präparate von oben nach unten durchmustert, so begegnet man vor Allem im Nivequ des kaudalen Endes des Septum pellucidum einer Wunde, welche als ein unregelmässig-dreieckiger Substanzdefekt im dorso-lateralen Teil des Claustrum wahrgenommen wird. Etwas kaudaler im Niveau des kranialen Eades des Ammonshorns tritt die Wunde als ein Y-formiger Schlitz im dorsalen Teil des Putamens zutage, wovon unmittelbar lateral noch 2 ovale Wunden angetroffen werden. Noch weiter kaudal im Niveau des Anfangsteils des Ammonshorns zeigt das Putamen weiter noch 2 ziemlich bedeutende Wunden, eine dicht an der inneren Kapsel anliegende (Fig. 5, La) und eine ventral davon im ventralen Putamenteil angetroffene (Fig. 5, Lb). Die letztere findet sich dicht an der äusseren Kapsel, ist etwas grösser als die erstere und hat eine unregelmässige Kontur, während die erstere mehr rundlich ist. Die beiden Wunden sind durch einen schmalen Isthmus miteinander verbunden, welcher auf den Schnitten brückenartig zutage tritt (Fig. 5, Lc). Weit kaudal etwa im Niveau des kranialen Endes des Sehhügels weist auch der Globus pallidus eine leichte, gabelformig erscheinende Wunde in seiner dorso-lateralen Partie auf, während die innere Kapsel und das Putamen in dieser Hóhe ganz intakt bleiben.

Nach der Erwähnung der durch die Operation bedingten Wunde bleibt es nun übrig, die Nissl-Degeneration in Nucleus caudatus, Globus pallidus und Putamen auseinanderzusetzen. Weit kranial von den Wunden des Putamens ungefähr im Niveau des kranialen Endes des Septum pellucidum treten die grossen Zellen des Nucleus caudatus auf der operierten Seite meistens degeneriert zutage (Fig. 6). Die degenerierten Zellen sehen aufgebläht und homogen aus; ihr Kern zeigt sich undeutlich konturiert und manchmal intensiver als beim normalen Zustande gefärbt. Auch vermag man die exzentrische Verlagerung des Kernes und das Verschwinden des Kernkörperchens nachzuweisen. Was den Befund an den kleinen Zellen angeht, so fallen nur wenige von ihnen der Degeneration anheim. Auch an diesen degenerierten kleinen zeigt sich der Zellkorper aufgetrieben und blass; über die Oberfläche desselben tritt der Kern nach auasen hervor, welchen man auch manchmal gänzlich vermissen kann.

Im Niveau, wo das Putamen stark zerstort ist, wird nıan ausser den oben 'geschilderten degenerierten kleinen zwar noch einiger, pyramidenformig umgestalteter pyknotischer kleiner Zellen gewahr. Die letzteren finden sich aber nur im dorsalen Abschnitt des Nucleus caudatus beschränkt, während die ersteren sowohl im dorsalen wie im mittleren Teil desselben wahrgenommen werden. Noch weiter kaudal im Niveau, wo der Globus pallidus an seinem dorso-lateralen Abschnitt verletzt ist, nehmen die degenerierten grossen Zellen etwas an Zahl ab, wohingegen sich die degenerierten kleinen augenscheinlich etwas vermehren. 
Auf der nicht operierten Seite reagiert der Nucleus caudatus auf diè Operation so schwach, dass hier die Nissl-Degeneration nur in äusserst sp:̈rlichen grossen Zellen sich abspielt. Hier sei darauf aufmerksam gemacht, dass ich bei diesem Experimente ausserstande bin, irgend eine Wucherung des Gliagewebes austindig zu machen.

Im Globus pallidus wird die Nissl-Degeneration schon im Niveau des kranialen Endes desselben gefunden, wo das Putamen stark verödet ist. In dieser Höhe fallen nicht wenige Zellen des Globus pallidus der Degeneration anheim, und die degenerierten Zellen sind hauptsächlich im dorso-lateralen, zum kleinen Teil aber in ventro-medialen Abschnitt desselben anzutreffen. Im Niveau, wo der Globus pallidus an seinem dorsolateralen Abschnitt leicht verletzt ist, erweisen sich die Zellen meistens als degeneriert, und zwar in der Umgebung der Wunde tritt die Degeneration am ausgeprägsten zutage. Auch im Globus pallidus der nichtoperierten Seite begegnet man degenerierten Zellen; jedoch sind sie hier viel weniger zahlreich und viel leichter degeneriert, als im Ganglion der operierten Seite.

Das Putamen bietet schon etwas kranialer von seiner Wunde degenerierte grosse und kleine Zellen dar. Im Niveau, wo das Putamen ausgedehnt verletzt ist, werden bochgradig veränderte Zellen sowohl dorsal, als auch ventral von der Wunde wahrgenommen, und die Degeneration tritt hier stärker zutage als dort. In dieser Hőhe nehmen die degenerierten kleinen Zellen etwas an Zahl ab, und einige von ihnen erscheinen schmutżig gefärbt. Endlich im Niveau, welcher etwas kaudaler von der Wunde sich findet, betrifft die Degeneration nur spärliche grosse und kleine Zellen.

Was das Gliagewebe anbetrifft, so kann kranial von der verletzten Stelle von einer Wucherung desselben kaum die Rede sein. Dagegen gerät das Gliagewebe im Niveau der Wunde dorsal und ventral von derselben in eine mässige Wucherung. Die Gliawucherung nimmt kaudal von der lädierten Stelle wieder etwas an Intensität ab und ist nur in dem medialen Putamenteil nahe der Wunde des Globus pallidus anzutreffen.

Das Putamen der nicht operierten Seite bleibt im Allgemeinen von der degenerativen Veränderung verschont, nur dass hier spärliche grosse und kleine Zellen verändert sich zeigen.

Kurz gesagt, wird bei diesem Experimente das Putamen ungefähr im Niveau seines mittleren Drittels ausgedehnt verödet, wobei der Globus pallidus auch im Niveau seines mittleren Teils leicht mitverletzt ist. Wie oben dargetan, hat das Experiment die NisslDegeneration im Nucleus caudatus verursacht. Bei der Mitverletzung des Globus pallidus drängt sich zwar die Frage auf, ob die Nissl-Degeneration entweder auf die Verletzung des Putamens oder auf die des Globus pallidus zurückzuführen ist. Man wird sich aber leicht davon überzeugen, dass die im Nucleus caudatus gefundene Degeneration ihren Grund nicht in der Verletzung des Globus pallidus, sondern in der des Putamens hat, wenn man in Rïcksicht nimmt, dass die Wunde des Globus pallidus sehr klein und nur in der dorso-lateralen Partie desselben beschränkt ist. Ebenso ist die ịn Globus 
pallidus angetroffene Nissl-Degeneration wohl auf die Verletzung des Putamens zurückzuführen. Dass die dicht an den verletzten Stelle gefundene Nissl-Degeneration auf einem entzündlichen Prozess beruht, ist schon daraus ersichtlich, dass sie immer von einer Wucherung des Gliagewebes begleitet ist.

Nach der Auseinandersetzung des Nissl-Befundes erübrigt es nun den Befund an denjenigen Gehirnen zu erwähnen, welche aus den 21 Tage die Operation überlebten Tieren abgetragen und nach dem Marchi-Verfahren untersucht sind. Die Untersuchung der Marchi'schen Präparate weist vor allen Dingen darauf hin, dass im Niveau des kranialen Endes des Chiasma opticorum das Putamen ausgedehnt und hochgradig zerstört ist ; auch wird die Capsula interna in ihrer dorso-lateralen Partie in Mitleidenschaft gezogen (Fig. 7). In Niveau des kranialen Endes des Tractus opticus ist der mediale Teil des Putamens mit dem kranialeu Teil des Globus pallidus verletzt. Etwas kaudaler davou tindet man noch je eine kleine Wunde in dem sich an's Putamen stossenden Abschnitt der inneren Kapsel und in der Mitte des Globus pallidus. Abgesehen von der Umgebung der verletzten Stelle wird man in dewn dorsalen Teil der inneren Kapsel und dem dorso-lateralen Abschnitt des Nucleus caudatus degenerierter Fasern gewahr. Bezüglich der Marchi'schen Degeneration in letzteren fragt es sich, worin ihr Grund liegt. Meiner Meinung nach ist sie nicht auf die Verletzung der inneren Kapsel, sondern wohl auf die von Putamen und Globus pallidus zurückzuführen, da ich mich schon davon überzeugt habe, dass die Zerstörung des Nucleus caudatus eine deutliche Nissl-Degeneration in den beiden Ganglien zur Folge hat. Deswegen scheinen mir die im dorso-lateralen Teil des Nucleus caudatus gefundenen degenerierten Fasern nicht aus der inneren Kapsel, sondern aus Putanen und Globus pallidus zu stanmen. Sie laufen durch die inneren Kapsel hindurch, um den dorso-lateralen Teil des Nucleus caudatus zu erreichen. Auch diejenigen degenerierten Fasern, welche im Globus pallidus, insbesondere im kranialen Teil desselben, gefunden werden, sind wohl ebenfalls auf die Verletzung des Putamens zurückzufihren.

Auf der nicht operierten Seite kann man die Marchi-Degeneration zwar nicht in Nucleus caudatus, wohl aber in Putamen und Globus pallidus konstatieren.

\section{Versuch III. Zerstörung des Globus pallidus.}

Die Operation wird in folgender Weise ausgeführt: Nach der Trepanierung des Schädelknochens steckt man eine spitze Nadel entweder etwa $1 \mathrm{~cm}$ lateral oder dicht lateral von der Fissura longitudinalis ins hintere Ende des Frontallappens. Ventromediale oder ventro-laterale Richtung einschlagend geht man durch die Hirnrinde hindurch in die Tiefe, um den rechten Globus pallidus zu zerstören. Dem operativen 
Eingriffe sind im Ganzen 3 Kaninchen unterworfen, deren Gehirne alle nach der Nissl'schen Methode untersucht sind.

Die Untersuchung der Präparate zeigt zunächst, dass bei einem Versuchstiere im Niveau des mittleren Drittels des Septum pellucidum der dorso-laterale Abschnitt der Capsula interna leicht verletzt ist. In dieser Höhe kann aber noch von der Nissl'schen Degeneration in Nucleus caudatus und Putamen kaum die Rede sein. Etwas kaudaler im Niveau des kaudalen Endes des Septum pellucidum ist eine starke Destruktion des Globus pallidus nebst einer leichten Mitverletzung der Capsula interna bemerkbar (Fig. 8). Noch weiter kandal ist dẹr Globus pallidus in seinem an's Putamen angrenzenden Abschnitt ziemlich stark verletzt, während die Capsula interna und das Putamen in dieser Höhe vom degenerativen Prozess verschont bleiben. Das beim 1. Versuchstiere gesagte gilt im wesentlichen auch für die übrigen, so dass es mir überflüssig scheint, die Befunde der einzelnen wiederholt anzugeben.

- Im Nucleus caudatus werden die grossen Zellen meistens degeneriert gefunden, und eine Anzabl von den degenerierten Zellen zeigen sich eingeschrumpft und pyknotisch gefärbt, während die anderen eine Aułblähung des Zellkorpers und eine exzentrische Verlagerung des Zellkernes infolge der Auflösung der Nissl'schen Körper darbieten. Jedoch ist es kaum moglich, hier irgend eine Wucherung des Gliagewebes und eine Veränderung der kleinen Zellen ausfindig zu machen. Im Gegensatz dazu zeigt sich das Gliagewebe in demjenigen Putamenteil etwas gewuchert, welcher an die Wunde des Globus pallidus unmittelbar sich anschliesst. Hier erweisen sich die grossen und kleinen Zellen als degeneriert; insbesondere nehmen die kleinen etwas an Zahl ab und treten schmutzigblass gefärbt auf. Im übrigen Abschnitt des Putamens erhalten die kleinen Zellen aber ihr normales Aussehen aufrecht, während die grossen meistens in Degeneration verfallen und ganz dieselben Degenerationsbilder aut'weisen, wie die des Nucleus caudatus.

Das Resultat spricht ohne Weiters dafür, dass die aus den grossen Zellen ron Nucleus caudatus und Putamen entspringenden Fasern in den Globus pallidus eintreten.

Bei dieser Gelegenheit sei hinzugefügt, dass ich mich in meiner vorigen Arbeit anch damit beschäftigte, die Marchi-Degeneration in dem Corpus striatum und den anderen Hirnteilen nach Zerstórung des Globus pallidus zu untersuchen. Dabei konnte ich diejenigen Fasern konstatieren, welche aus dem Globus pallidus stammen und teils durch die innere Kapsel bindurch in den Nucleus caulatus, teils direkt in's Putamen eintreten.

Wie aus dem Versuch I hervorgeht, erweisen sich die Zellen des Putamens, namentlich die grossen, bei Zerstörung des Nucleus caudatus als veründert. Ausserdem nimmt man beim Versuch II wahr, dass die Zerstorung des Putamens wohl die Marchi-Degenera-

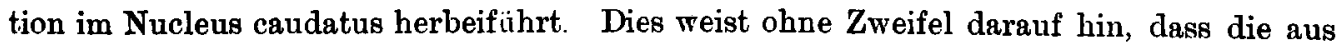


den Zellen des Putamens, insbesondere aus den grossen, entspringenden Fasern in den Nucleus caudatus eintreten, wie Wilson zutreffend angibt.

Nach Bielschowsky; Vogt und Anderen sollen die kleinen Zellen des Putamens nur Schaltelemente representieren. Meiner Meinung nach müssen auch die kleinen Zellen des Putamens wenigstens zum Teil ibren Axon in den Nucleus caudatus hineinschicken, wie die grossen; denn die ersteren erweisen sich auch nach Zerstörung des Nucleus caudatus wenn auch nur spärlich, so doch sicherlich als degeneriert.

Ferner geraten zahlreiche Zellen des Globus pallidus infolge der Zerstorung des Nucleus caudatus in Degeneration. Früher war ich ausserstande, an der Hand der IIarchi'schen Präparate diejenigen Fasern nachzuweisen, welche nus dem Globus pallidus stammend durch die innere Kapsel hindurch in den Nucleus cauclatus eintreten. Da die Fasern jetzt wohl durch den Versuch III im Einkrang mit Probst, Grünstein, Minkowsky und Anderen konstatiert werden, so liegt es auf der Hand, dass der Nucleus caudatus auch die aus dem Globus pallidus stammenden Fasern enthält.

Aus dem Versuch $\Pi$ ergibt sich, dass auf die Zerstorrung des Putamens nicht wenige Zellen des Nucleus caudatus reagieren. Dementsprechend bestätigt der Versuch I, dass die Verletzung des Nucleus caudatus die Marchi-Degeneration im Putamen herbeiführt. Daher unterliegt es keinem Zweifel, class die aus dem Nucleus caudatus entspringenden Fasern zum Teil nach dem Putamen streben, um bier zu endigen. Die Fasern sind aber kurz und vielleicht von einer gauz dünnen Markscheide umbüllt, so dass ich früher sie reimisste.

Die Nissl'sche Veränderung in Nucleus caudałus nach Zerstórung des Globus pallidus (Versuch III) und die Marchi-Degeneration im Globus pallidus nach Verletzung des Nucleus caudatus (Versuch I) erlauben uns diejenigen Fasern zu ermitteln, welche dem Nucleus caudatus entstanmend in den Globus pallidus eintreten, um sich hier zu zersplittern. Die Fasern wurden zwar schon in meiner vorigen Arbeit angegeben; damals war es aber unmoglich zu entscheiden, ob sie im Globus pallidus endigen oder .nur durch denselben hindurch passieren, um weiter kaudalwärts zu ziehen.

Die Nissl-Degeneration im Putamen nach Zerstorung des Globus pallidus (Versuch III) und die Marchi-Degeneration in Globus pallidus nach Verletzung des Putamens (Versuch II) sprechen für das Existieren derjenigen Fasern, welche aus dem Putamen eutspringen und im Globus pallidus endigen. Gerade umgekhrt kann man aus der Nissl-Degeneration im Globus pallidus nach Verletzung des Putamens (Versuch $\Pi$ ) und aus der Marchi-Degeneration im Putamen nach Zerstorung des Globus pallidus (Tersuch III) wobl diejenigen Fasern erschliessen, welche im Globus pallidus ihren Ursprung nehmen und im Putamen sich zersplittern.

Wenn man das oben angegebene kurz zusammenfasst, so ergibt sich daraus, dass zwischen dem Nucleus caudatus, dem Putamen und lem Globus pallidus eine korrelative Beziehung besteht, indem die 3 genanten Ganglien miteinander die Fasern austauschen. 
Da die einseitige Zerstorung des Nucleus caudatus keine Degeneration des kontralateralen Kernes zur Folge hat, so ist es klar, dass zwischen den beiderseitigen Kernen keine Kommissurenfasern vorhanden sind.

Wenn auch beim Versuch $I$ spärliche veränderte Zellen in Putamen und Globus pallidus der nicht operierten Seite nach Verletzung des Nucleus caudatus gefunden werden, so lässt sich doch keine Marchi-Degeneration im kontralateralen Nucleus caudatus nach Zerstörung von Putamen und Globus pallidus nachweisen. Daher muss man Bedenken tragen solche Fasern anzunehmen, welche in Putamen und Globus pallidus entspringend in den kontralateralen Nucleus caudatus eintreten.

Trotz eịniger veränderter Zellen im kontralateralen Nucleus caudatus nach Zerstörung des Putamens (Versuch II) ist die Marchi-Degeneration im kontralateralen Putamen nach Verletzung des Nucleus caudatus (Versuch I) kaum nachweisbar; deshalb ist es auch sehr fraglich, ob es solche Fasern gibt, welche aus dem Nucleus caudatus stammend im kontralateralen Putamen sich zersplittern. Ebensowenig kann man sich auch von denjenigen Fasern überzeugen, welche aus dem Globus pallidus entspringend in dem kontralateralen Putamen und dem Globus pallidus endigen, wie es aus dem Versuch III ersichtlich ist.

Aus den oben geschilderten Tatsachen kann man wohl den Schluss ziehen, dass das Corpus striatum und der Globus pallidus einer Seite keine Fasern in die Kerne der gegenüberliegenden hineinschicken, so dass zwischen den beiderseitigen Kernen keinerlei Faseraustausch stattfindet.

Zum Schluss werde jch nicht unterlnssen, Herrn Prof. Dr. Kosnkn für seine freundliche Anregung und Leitung, Herrn Prof. Dr. Yugitu für seine freundliche Unterstïtzung bei dieser Arteit mejnen herzlichen Dink nuszusprechen.

\section{Literaturverzeichnis.}

1) Probst, U̇̉ber die Rinden-Sehhïgelfısseın des Riechfeldes, über das Gewölbe, die Zwinge, die Rındb.ogenfısern, über die Schweifkeınfusernng und über die Verteilnng der Pymmidenfasern im Pymmidenarenl. Arch. f. Anut. n. Physiol. Annt. Abteil. 1903. $\quad$ 2) Pollnk, Beitrng zur Pathologie der extrnpy mmidılen Bewegungsstörungen (U̇ber Wilsonєche Linsenkerndegenerntion). Zeitschr. f. d. ges. Neurol. u. Psycb., LXXVII. 3) Grïnstein, Zur Frage von den Leitungsbahnen des Corpus striatum. Neurol. Zentrnlbl. 1911, H. 12 . 4) Minkowsky, Etudio sur les connexions anutomiqes des circonvolutions rolnndiqes, puietules et frontules. Arch. Saisses de Neurol. et de Psychint. 1924. 5) Vogt, Zur Lehre der Erkrankungen des strï̈ren Systems. Journ. f. Psychol. u. Neurol. Bd. 25, 1920 . 6) Jnkob, Die extrapyrumidulen Erkrnnkungen mit besonderer Beı̈̈cksichtigung der pathologischen Anntomie 1. Histologie u. der Puthophysiologie der Bewegungsstörungen. 1923. 7) Wilson, An experimentul resenrch into the mutomy und physiology of the corpus strintum. Britu, Vol. 36, 8) Valkenburg, Experimental und pathologico-nnatomical reserrches on the corpus cullosum. Brnin, $1913 . \quad$ 9) Winklef 
nnd Potter, An anntomioul gujde to experimenitul reserurch on the mbit's brnin. 1911. 10) Inui, Über die Verbindung des Globus pallidus mit dem Corpus Lnyaj und der Substantin nigm beim Knninohen. Oknyamn Ignkknj-Zasshi. Jg. 39, Nr. 4 (Nr. 147), April 1927.

\section{Verzeichnis der Abkürzungen.}

C. ext. = Cupsıla externı.

DZ. = Degenerierte Zellen.

DklZ. = Degenerierte kleine Zellen.

Lu. = Inesionsstelle a im Putamen.

Irc. $=$ Iacsionsstelle c im Pntamen.

NZ. $=$ Normule Zellen.

Bep. pell. = Septum pellnoidum.
C. int. = Crpsuln internn.

DgrZ. = Degenerierte grosse ZeПlen.

$I_{\text {. }}=$ Iäsionsstelle.

IJ.. = Lnesjonsatelle b im Putrmen.

N. C. oler N. Ound. = Nucleus cundutus.

Put. = Putnmen.

Ven. lut. $\boldsymbol{\Rightarrow}$ Ventrioulus luternlis.

\section{Erklärung der Abbildungen,}

Fig. 1. Inesionsstelle des rechten Nuclens cnndutus in seinem mittleren Teil.

Fig. 2. Degenerierte grosse und kleine Zellen des Putnmens infolge der Ve، letznng des Nucleus candatus (Nissl-Methode). Operntjonssejte. Versuch I.

Fig. 3. Degenerierte Zellen des Globus pnllidus nach Zerstörnng des Nuclens onudntus (Nissl-Methode). Opemtionsseite. Versmch I.

Fig. 4. Lnesion des reohten Nucleus candntus, und Degenerution im Nucleus onudutus und Putnmen nnf der operierten Seite (Murchi-Methode). Versuch J.

Fig. 5. Verletzungsstelle des rechten Putnmens (Nissl-Methcde). Versuch II.

Fig. 6. Degenei ierte grosse Zellen des Nucleus cnndntus infolge der Verletzung des Putumens (NisslMethode). Operntionsseite. Versuch $\Pi$.

Fig. 7. Lnetionsstelle des rechten Putnmens, und Degenerution im Nucleus cnndntus (Mnrchi-Methode). Versuch .II.

Fig. 8. Verletzungsherd des rechten Globus pallidus in seinem dorso-lnternlen Tejl (Nissl-Methode). Versuch III.

\section{內 容大意}

\section{民狀核，被钤及ヒ淡蔡球間ノ相互關係二就テ}

\section{岡山哥科大舁解剖學敌室（主任上坂致报）}

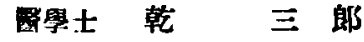

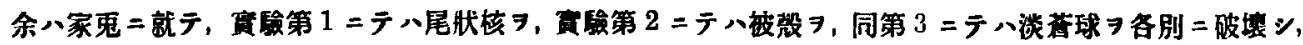

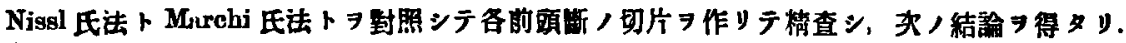


Fig. 1.

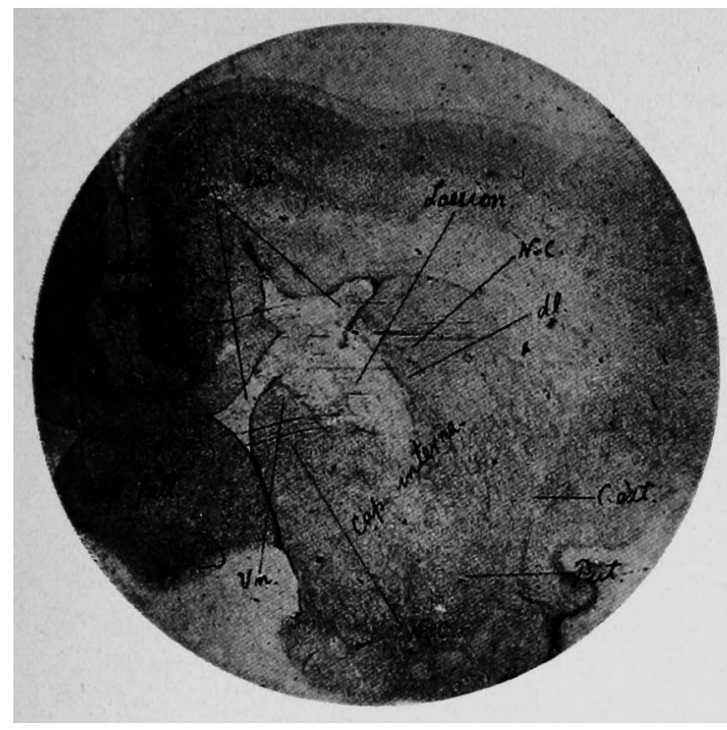

Fig. 3 .

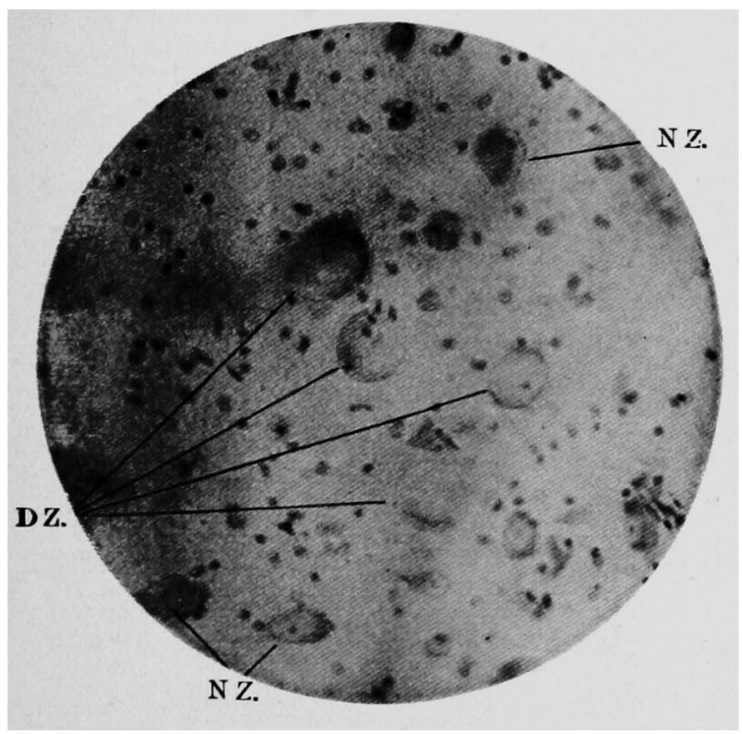

Fig. 2.

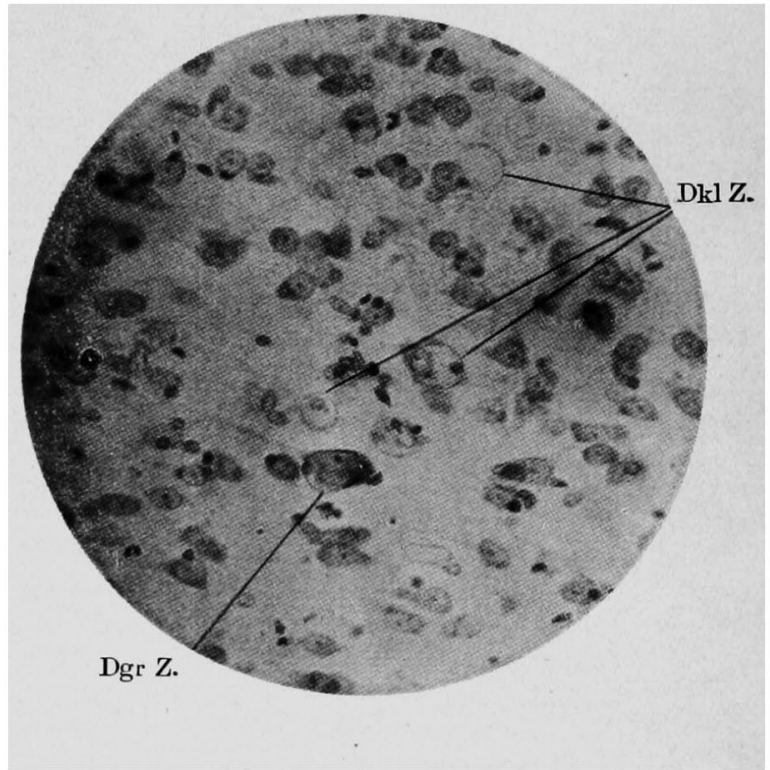

Fig. 4.

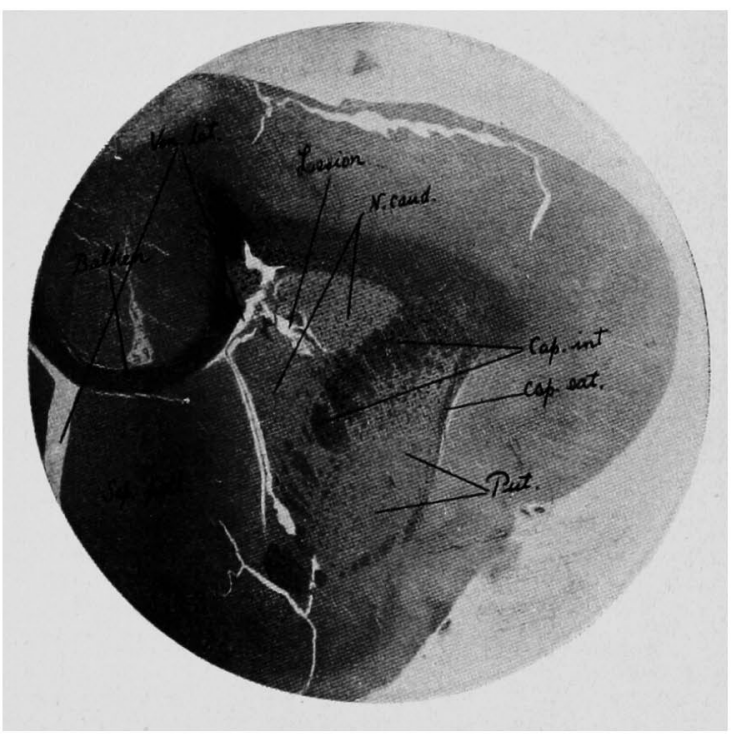

Saburo Inui-Ueber die korrelativen Beziehungen zwischen dem Nudeus caudatus etc. 
Fig. 5.

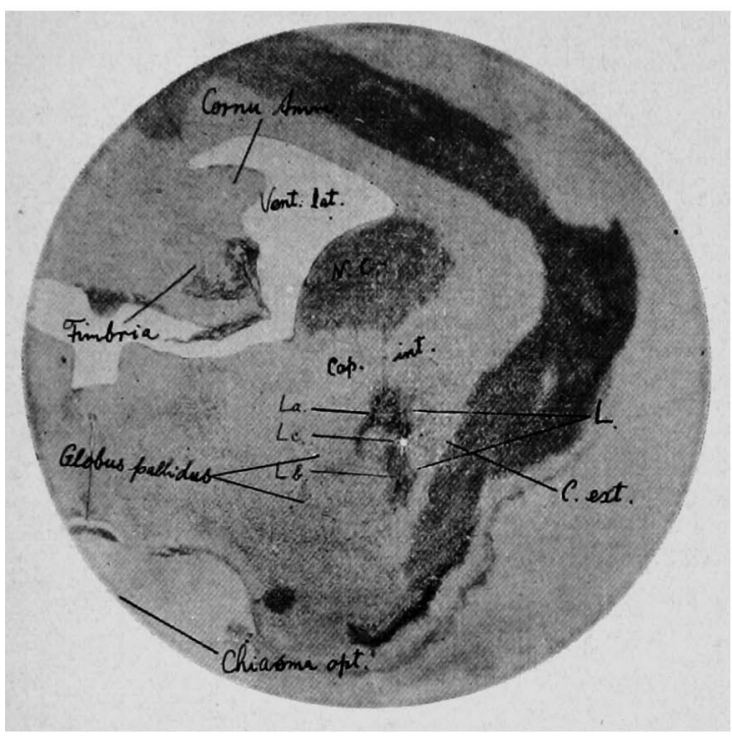

Fig. 7.

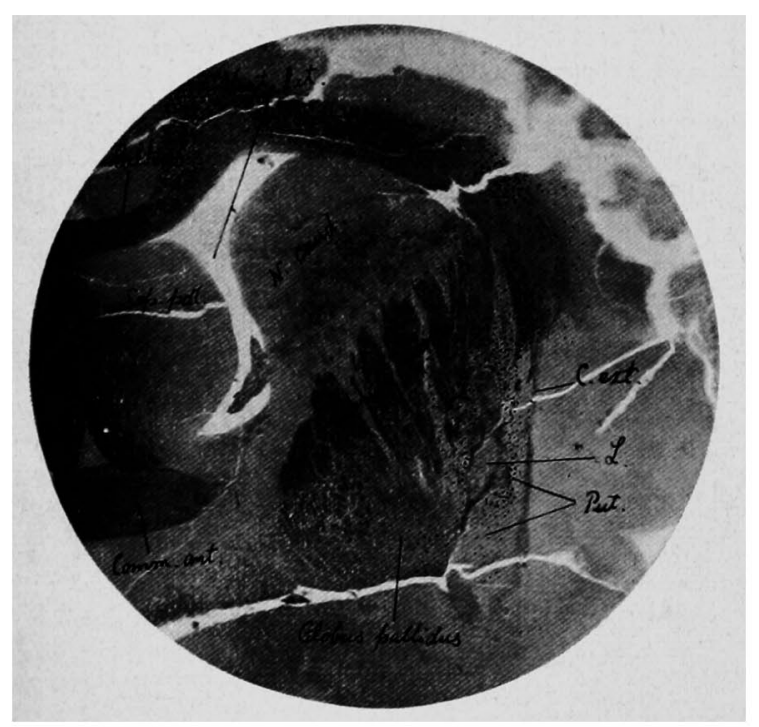

Fig. 6.

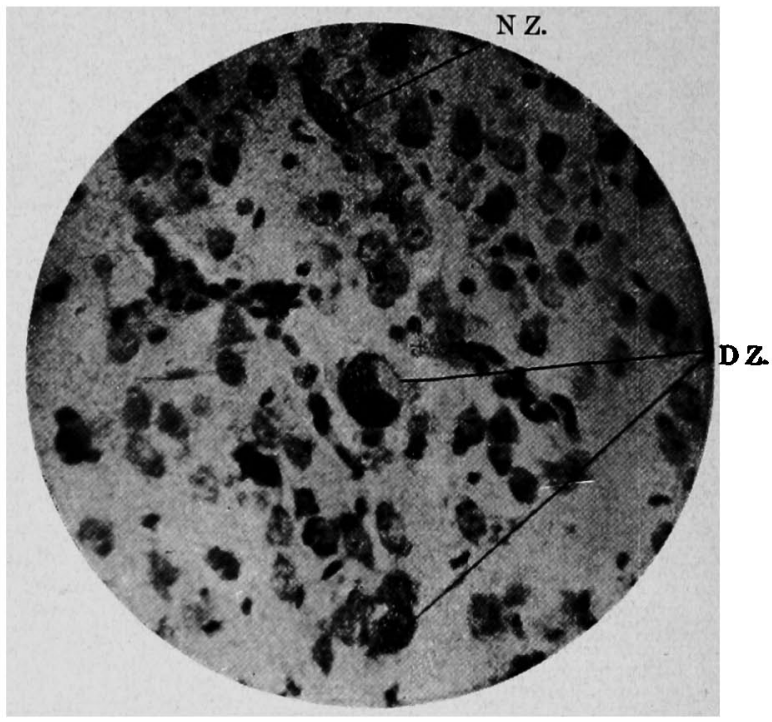

Fig. 8.

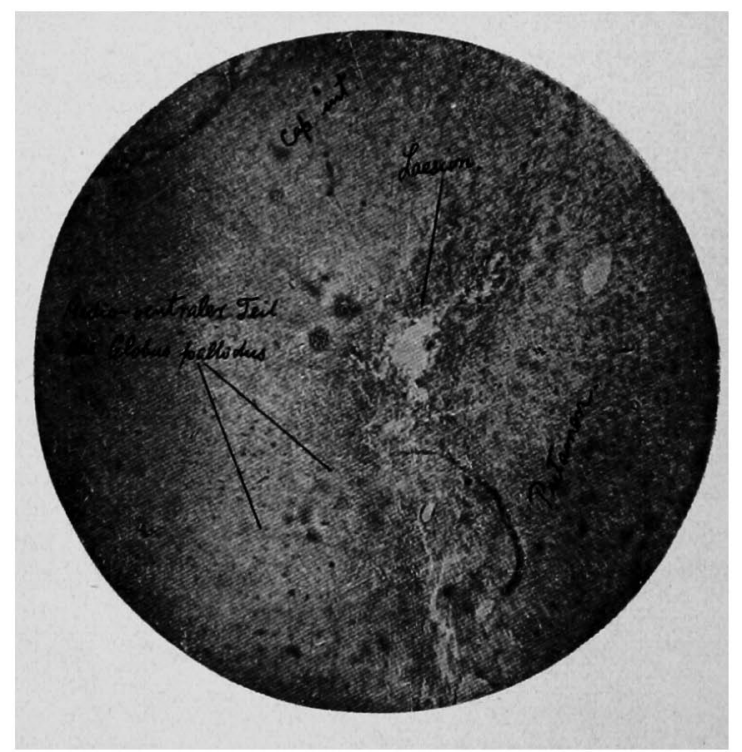

:Saburo Inui-Ueber die korrelativen Beziehungen zwischen dem Nadeus caudatus etc. 


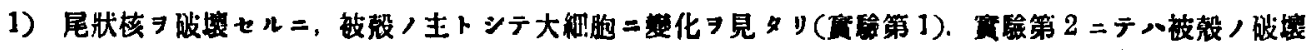

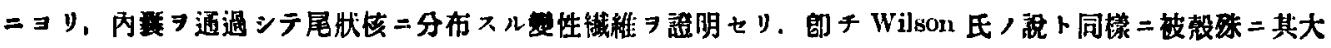

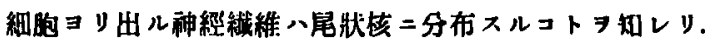

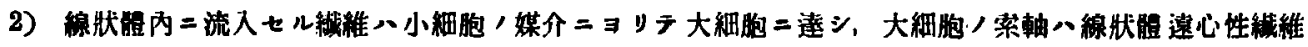

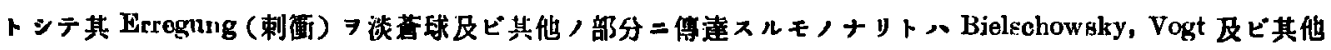

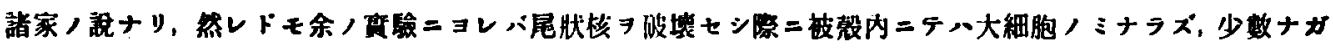

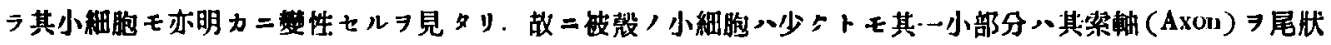

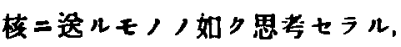

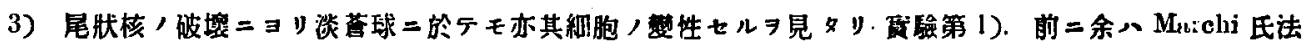

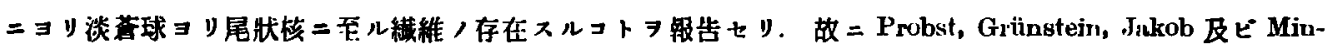

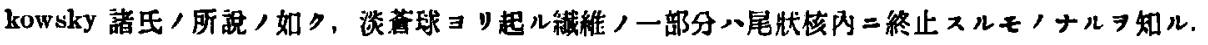

4) 次二被敖

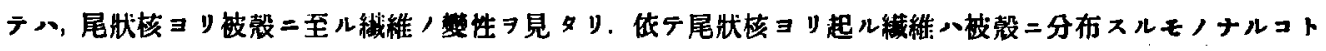
ヨ知レリ.

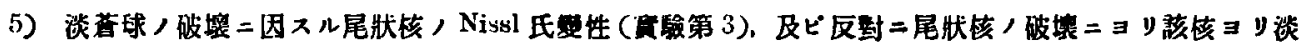

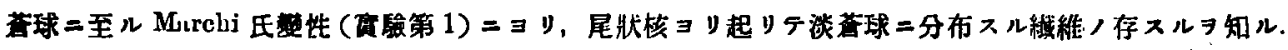

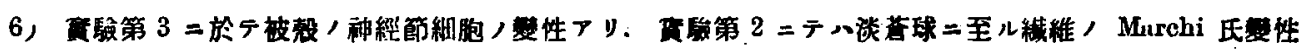

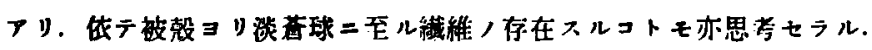

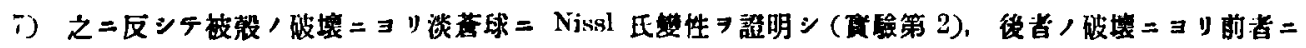

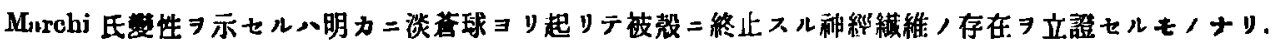

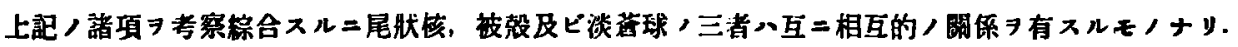

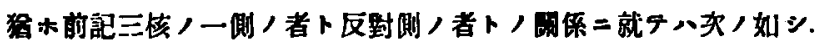

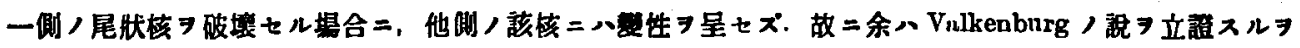

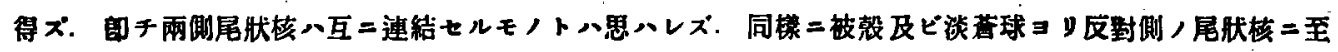

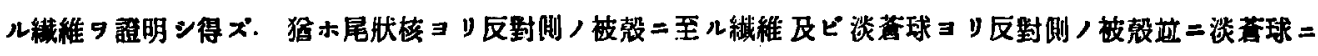
至几栱維 $モ$ 證明スル

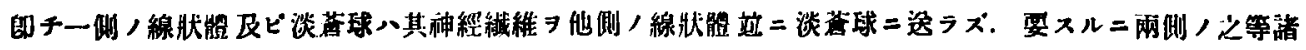

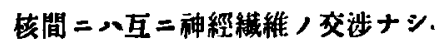

